\title{
Role of Endobronchial and Endoscopic Ultrasound in Pulmonary Medicine
}

\author{
D. Gompelmann F.J.F. Herth
}

Pneumology and Critical Care Medicine, Thoraxklinik at University of Heidelberg, and Translational Lung Research Center Heidelberg, Member of the German Center for Lung Research, Heidelberg, Germany

\section{Key Words}

Endobronchial ultrasound · Esophageal ultrasound-guided fine needle aspiration · Pulmonary lesion - Transbronchial needle aspiration

\begin{abstract}
In the last decade, endobronchial ultrasound (EBUS) has evolved into an important tool for diagnostic bronchoscopy in daily practice. EBUS has established as a standard for the diagnosis of hilar or mediastinal lymphadenopathy, as a guidance technique for peripheral pulmonary lesions and for the evaluation of tumor involvement of the tracheobronchial wall or mediastinum. In the meantime, EBUS has also taken a significant role in mediastinal staging in lung cancer patients. However, EBUS plays not only a role in the diagnosis of malignancies, it is also important for the diagnosis of benign disorders.

(c) 2013 S. Karger AG, Basel
\end{abstract}

\section{Introduction}

Endosonography was initially introduced in the field of gastroenterology, where it was preferentially used for the staging of carcinomas of the esophagus, cardia and colon-rectum and thus affected tumor therapy [1]. In the 1990s, endosonography was developed for the diagnosis of airway diseases. The main problem that had to be solved was to make contact between the probe and the transbronchial wall. Thus, ultrasound probes with a water-fillable balloon at their distal tip were introduced providing a complete $360^{\circ}$ view and thus allowing visualization of the airway wall and parabronchial structures. Nowadays, endobronchial ultrasound (EBUS) is a routinely performed procedure and plays a role in

- early lung cancer staging;

- the evaluation of tumor infiltration of the tracheobronchial wall and mediastinum;

- the diagnosis of peripheral pulmonary lesions, and

- in the diagnosis of hilar-mediastinal lymphadenopathy and mediastinal staging in lung cancer patients.

Thereby, different techniques of EBUS and various probes are available. The first EBUS probes were described by Hürter and Hanrath [2, 3] in 1990 and 1992. The latest development, however, is the EBUS scope that provides visualization of mediastinal masses and enlarged lymph nodes and allows a real-time transbronchial needle aspiration (TBNA).

\section{Early Lung Cancer Staging}

Early lung cancer is defined as roentgenographically occult but endoscopically visible cancer with a surface area $<2 \mathrm{~cm}$ and no invasion beyond the bronchial carti-

\section{KARGER}

E-Mail karger@karger.com

www.karger.com/res
(C) 2013 S. Karger AG, Basel

0025-7931/13/0871-0003\$38.00/0
Dr. med. D. Gompelmann

Pneumology and Critical Care Medicine, Thoraxklinik Heidelberg Amalienstrasse 5

DE-69126 Heidelberg (Germany)

E-Mail daniela.gompelmann@med.uni-heidelberg.de 
lage [4]. In the last decades, diagnosis of early lung cancer was improved by the introduction of autofluorescence imaging (AFI) [5] and narrow band imaging (NBI) [6]. Up to date, the therapy of choice is still surgical resection. A limitation of surgery, however, is that in most cases significant normal lung parenchyma has to be removed and therefore prohibits surgical intervention in many patients. For these patients, a curative endobronchial treatment is available. Prior to endoscopic intervention, an accurate determination of the depth of endobronchial tumor lesions is crucial and influences therapeutic decisions. Since endobronchial therapeutic options including photodynamic therapy, electrocautery or cryotherapy cannot penetrate the exterior wall of cartilage, tumors with extracartilaginous growth cannot be treated bronchoscopically with curative intent [4].

The use of high-resolution computed tomography (HRCT) enables the evaluation of extrabronchial tumor spread and lymph node involvement [7] but cannot clarify carcinoma invasion of the different layers of the airway wall. As EBUS visualizes the 7-layer sonographic structure of the airway wall, including the mucosa, submucosa, endochondrium, cartilage, perichondrium, connective tissue and adventitia [8], it allows identifying small tumor lesions and evaluating the tumor depth. Therefore, a 20$\mathrm{MHz}$ mechanical radial ultrasound probe with a waterfillable balloon is introduced through the working channel of a conventional flexible bronchoscope. After filling the balloon with water, a contact to the airway wall can be achieved providing a $360^{\circ}$ view of the airway wall and parabronchial structures.

Prospective studies demonstrated that EBUS provides a quite accurate tool to analyze the extent of early lung cancer lesions. One important study was published by Miyazu et al. [9]. In 18 patients with squamous cell cancer lesions, EBUS was used to assess intrabronchial invasion. Nine patients, in whom intracartilaginous tumor lesions were observed by EBUS, underwent photodynamic therapy. These patients were disease free during a median follow-up of 32 months. In the remaining 9 patients, extracartilaginous tumor extent was detected. Six of 9 nine patients underwent surgical resection. Histological evaluation confirmed the extracartilaginous tumor infiltration in all these patients. Another trial also demonstrated the effective use of EBUS in the assessment of tumor extent. In 22 patients with early lung cancer, the sensitivity, specificity and positive predictive value of EBUS in evaluating carcinoma invasion within the bronchial cartilage was $85.7,66.7$ and $85.7 \%$, respectively [10].
By evidence or the exclusion of airway wall infiltration, EBUS also helps to precisely characterize lesions that were observed under AFI. Destruction of the 7-layer sonographic structure or thickening of the airway wall is for example an indication for malignancy. Using AFI alone, malignancies were correctly diagnosed in $69 \%$ in a 2003 published trial. Combining AFI and EBUS, malignant lesions were correctly diagnosed in 97\% [11].

\section{Evaluation of Tumor Infiltration of the Tracheobronchial Wall and Mediastinum in Advanced Cancer}

The evaluation of airway wall invasion is not only important in case of early lung cancer, but also in advanced lung cancer, particularly in patients who are considered to undergo surgery. Also, in these cases, the radial EBUS probe allows to determine tumor infiltration of the tracheobronchial wall, mediastinum, vessels or esophagus. EBUS even differentiates between airway infiltration and airway compression by tumor and thus has a marked influence on therapeutic decisions. Regarding the evaluation of airway involvement of intrathoracic bronchogenic tumors or metastatic diseases, EBUS is clearly superior to CT with an accuracy of 94 versus 51\% [12]. Another prospective trial compared EBUS, computed tomography (CT) and magnetic resonance imaging (MRI) for the evaluation of tracheobronchial wall infiltration in 54 patients with esophageal or thyroid cancer. The final diagnosis was based on histological results following surgical intervention and/or clinical findings. Sensitivity and specificity of EBUS was 83 and $92 \%$ and thus notably superior to those of CT (56 and 59\%) and MRI (73 and 75\%) [13].

\section{Diagnosis of Peripheral Pulmonary Lesions}

Peripheral pulmonary lesions still represent a challenge in the daily routine of the interventional pneumologist. Transbronchial biopsy (TBB) under fluoroscopic guidance is regarded as the standard procedure for the diagnosis of pulmonary nodules but it is often associated with a poor diagnostic yield.

However, since the introduction of ultraminiature radial ultrasound probes, the diagnosis of peripheral nodules was considerably improved, and thus EBUS is now firmly established in the guidelines. According to the guidelines of the American College of Chest Physicians, 
the use of EBUS is recommend in patients with peripheral pulmonary lesions in whom tissue diagnosis is required due to uncertainty of diagnosis or poor surgical candidacy [14].

The insertion tube of the ultraminiature radial ultrasound probe has a narrow diameter of 1.4 or $1.7 \mathrm{~mm}$ and thus can be introduced through the working channel of a standard bronchoscope. As the peripheral airways have a very small diameter, a water-fillable balloon at the distal end of the probe is not necessary to establish contact with the airway wall. Using a frequency of $20 \mathrm{MHz}$, a high resolution and detailed imaging of the peripheral lesions can be obtained [15].

The ultrasound probes can be used as guidance system for TBB. Thereby, the probe is inserted into the airways where the pulmonary nodule is suspected. If the probe is advanced to the airways surrounded by the nodule, the image of a solid mass appears - darker and defined by a bright border (fig. 1) - which replaces the 'snowstormlike' whitish ultrasound image that is produced by normal ventilated lung parenchyma. After detection of the lesion and marking of the localization of the ultrasound probe, the probe is removed and various biopsy tools can be used for lesion sampling. There are two different options to mark the localization of the EBUS probe and thus of the target lesion. The most common approach is to perform EBUS guidance under fluoroscopic control that allows the visualization of the probe in the peripheral lung tissue. Alternatively, a guide sheath can be used as extended working channel in which the ultrasound probe and - after detection of the target - the biopsy tools can be inserted.

The first study related to EBUS-guided TBB in patients with peripheral lesions was reported in 2002 . In 50 patients with peripheral lesions, TBB were performed under sequential fluoroscopic and EBUS guidance. Whereas under fluoroscopic guidance TBB led to a diagnosis in $76 \%$, EBUS-guided TBB provided a diagnosis in $80 \%$. Even though the diagnostic yield of these two methods did not distinguish significantly, there was a trend for EBUS-guided TBB to be better for lesions $<3 \mathrm{~cm}$ in diameter [16]. In the last years, several further trials evaluating the efficacy of EBUS-guided TBB were performed and summarized in a meta-analysis that was published by Steinfort et al. [17] in 2011. Sixteen trials covering 1,420 patients undergoing EBUS-guided TBB to assess peripheral pulmonary lesions were analyzed. A point specificity of 1.0 and a point sensitivity of 0.73 of EBUS-guided TBB for the detection of malignancy were obtained. Furthermore, subanalysis of this study demonstrated that the le-

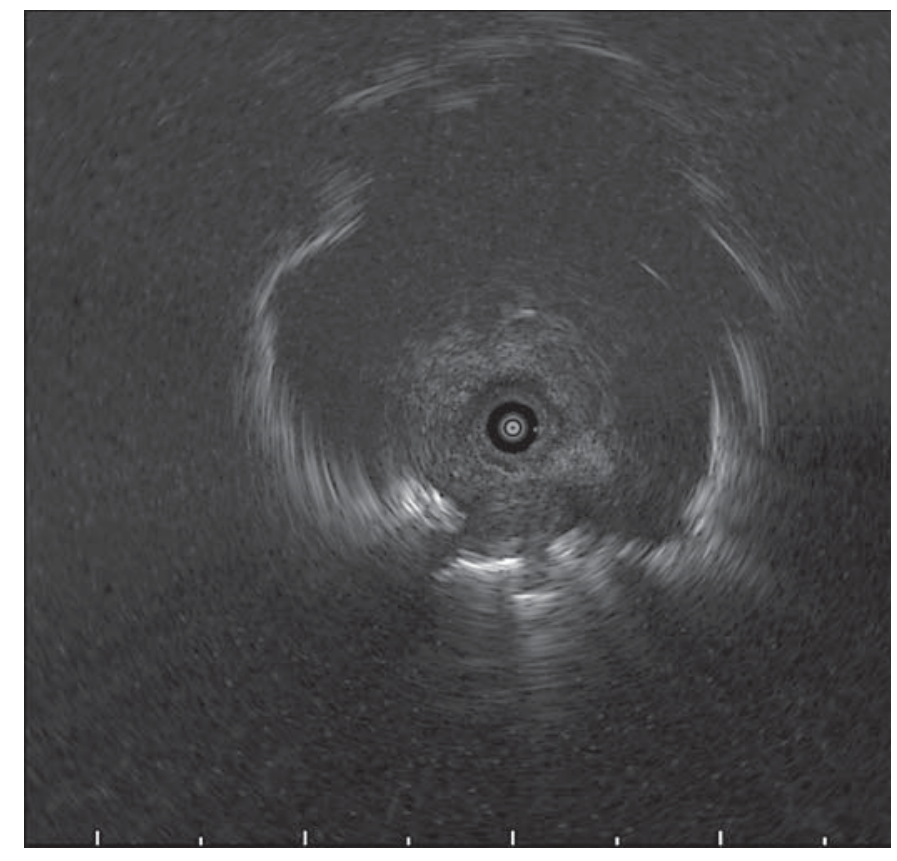

Fig. 1. Ultrasound image of a solid peripheral lesion.

sion size is an important factor affecting sonographic visualization. For lesions $\leq 20 \mathrm{~mm}$ in diameter, a diagnostic yield of $56.3 \%$ was observed, for lesions $>20 \mathrm{~mm}$, the diagnostic yield was $77.7 \%$. The correlation between lesion size and diagnostic yield could even be confirmed by a further trial. Furthermore, the final diagnosis as well as the distance from the lesion to the hilum also influences the EBUS visualization yield. Lesions $\geq 2 \mathrm{~cm}$, malignant lesions and a lesion to hilum distance $\leq 5 \mathrm{~cm}$ are associated with a higher visualization yield [18].

Due to the technological advancement and the development of further guidance techniques, one is continuously tempted to improve the diagnostic yield of EBUSguided TBB for peripheral pulmonary lesions. Nowadays, different bronchoscopic navigation systems are available that enable visualization of the peripheral lesion under real-time conditions. Asahina et al. [19] examined the efficacy of TBB using EBUS with a guide sheath combined with virtual-bronchoscopic navigation for the assessment of small peripheral lesions. Following successful virtualbronchoscopic navigation, the EBUS probe was advanced into the identified airway. In $80 \%$, the peripheral pulmonary lesions were sonographically visible and TBB could be performed. The diagnostic sensitivities of this combined approach were $44 \%$ for lesions $<2 \mathrm{~cm}$ and $92 \%$ for lesions from 2 to $3 \mathrm{~cm}$. 


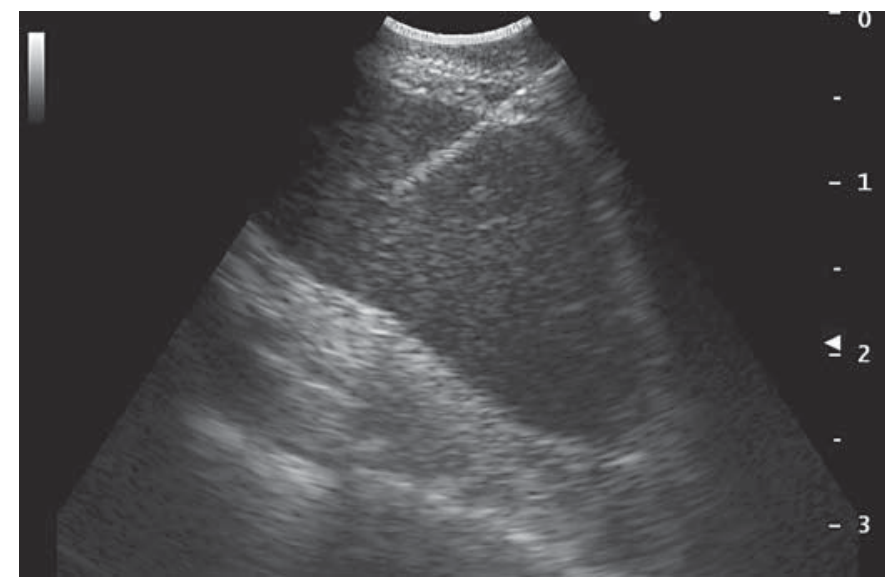

Fig. 2. EBUS-TBNA of a lymph node in position 7.

Another approach to increase the diagnostic yield of TBB for the assessment of peripheral lesions even further is the EBUS-guided cryobiopsy that provides a larger biopsy size [20]. Thirty-one patients with a peripheral lesion $<4 \mathrm{~cm}$ underwent sequentially EBUS-guided TBB with forceps as well as with the cryoprobe. The overall diagnostic yield of the lesions that were visualized with EBUS was $74.2 \%$. Comparing the EBUS-guided TBB by forceps vs. cryoprobe, a diagnosis could be established using the forceps in $61 \%$ and using the cryoprobe in $74 \%$.

\section{Diagnosis of Hilar-Mediastinal Lymphadenopathy and Mediastinal Staging in Lung Cancer Patients}

There are many reasons for hilar and mediastinal lymphadenopathy including benign as well as malignant diseases. Sarcoidosis, tuberculosis (TB) or unspecific inflammatory processes as well as metastases of various cancers or lymphomas can cause lymph node enlargement. Bronchoscopic assessment of lymph nodes by TBNA has already been performed using a rigid bronchoscope in 1949 [21]. In 1979, flexible needles were introduced allowing TBNA using a flexible bronchoscope [22]. Four years later, Wang and colleagues reported about TBNA for lung cancer staging, which is up to date the most frequent indication for TBNA [23]. The greatest progress in hilar and mediastinal lymph node assessment was finally the development of the EBUS scope that provides a real-time ultrasound-guided TBNA (EBUS-TBNA). This technique allows an accurate evaluation of lymph node involvement in lung cancer patients that is fundamental for therapy planning and determines survival.

This ultrasound puncture bronchoscope has a 7.5$\mathrm{MHz}$ convex transducer at its distal tip that provides ultrasound images via the contact of the probe with the tracheobronchial wall. Thus, enlarged lymph nodes or solid masses can be detected. Using Doppler imaging, blood vessels can also be shown. At the proximal end of the bronchoscope, a dedicated 21- or 22-gauge needle surrounded by a flexible sheath and containing an inner stylet can be advanced through the 2.2-mm channel. The needle can be inserted into the suspicious lesion under simultaneous ultrasound visualization. Afterwards, the inner stylet is removed and negative pressure is applied at the distal end, and the needle can be passed back and forth within the target lesion (fig. 2). The suction is then removed and the needle is withdrawn in the flexible sheath. Afterwards, cytological smears can be prepared or the specimen can be placed in formalin for cell block preparation [24].

By the introduction of this EBUS-guided TBNA, lymph nodes in positions 2, 4, 7, 10 and 11 can be assessed. In combination with esophageal ultrasound-guided (EUS) fine needle aspiration (FNA) using a linear EUS-FNA scope, lymph nodes in position 5, 8 and 9 can also be examined. The technique of EUS-FNA is very similar to that of EBUS-TBNA. The ultrasound transducer at the distal end of the EUS scope is pressed on the mucosa of the esophagus to obtain ultrasound images. The needle aspiration technique is similar to that of EBUS-TBNA. Besides the assessment of enlarged lymph nodes, EUSFNA enables the detection of metastases of the adrenal glands and the liver, too. As the examination of the left adrenal gland has already been well established (fig. 3), Uemura et al. [25] recently also reported the successful evaluation of the right adrenal gland. EUS-FNA can not only be performed using a special EUS scope, it can also be performed using only one linear EBUS scope in one setting $[26,27]$.

Up to date, these ultrasound-guided needle techniques (EBUS-TBNA and/or EUS-FNA) are recommended as the best first test in lung cancer patients with suspicious lymph node enlargement [28]. This recommendation in the guidelines of the American College of Chest Physicians is based on different meta-analyses. One of these meta-analyses summarized 26 studies reporting on 2,756 lung cancer patients who underwent EBUS-TBNA for mediastinal staging [28]. The median sensitivity of EBUSTBNA was $89 \%$, with values ranging from 46 to $97 \%$. The combination of EBUS-TBNA and/or EUS-FNA provid- 


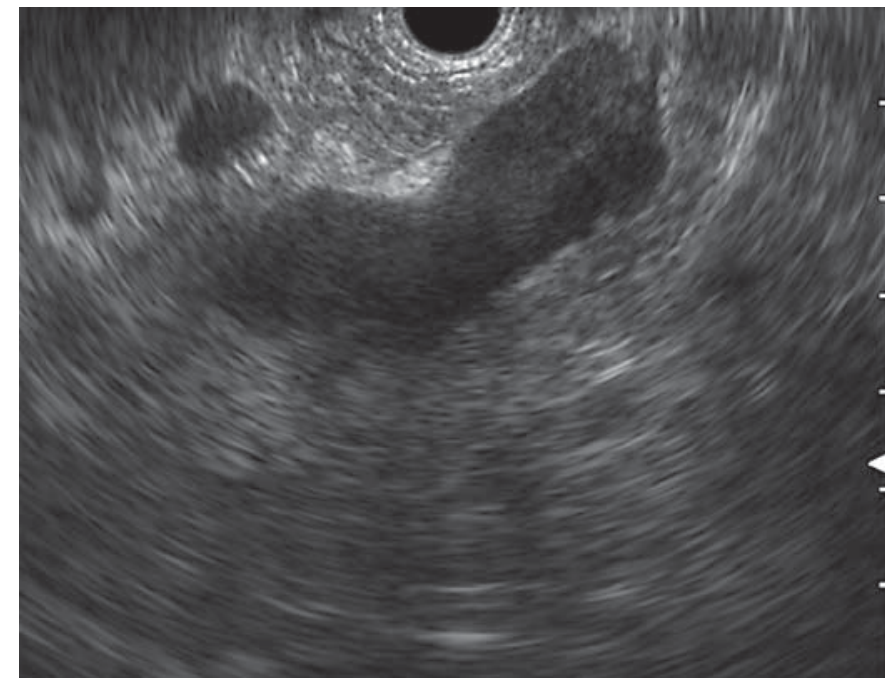

Fig. 3. Ultrasound image of the left adrenal gland (obtained by EUS scope).

ing a complete mediastinal staging in patients with lung cancer was examined in another meta-analysis covering 7 studies with 811 patients. The results revealed a pooled median sensitivity of $91 \%$ and a specificity of $100 \%$ [28].

Besides the efficacy studies, comparative effectiveness trials were performed demonstrating that the ultrasoundguided needle techniques are preferable to imaging techniques like CT or positron emission tomography [29] and also preferable to a primary mediastinoscopy $[30,31]$. A mediastinocopy is only recommended if EBUS-TBNA/ EUS-FNA revealed a negative cytological result in patients with suspicion of N2, N3 involvement (pathologically enlarged lymph nodes in CT or FDG-enhancing lymph nodes, central tumors or N1 lymph node enlargment) $[28,32]$.

EBUS-TBNA presents a useful diagnostic tool not only for malignant diseases but also for benign disorders. Diseases such as sarcoidosis or TB can be diagnosed by EBUS-TBNA. One recently published meta-analysis summarized 15 prospective and retrospective studies in which 553 patients with sarcoidosis underwent EBUSTBNA. The overall diagnostic yield was $79 \%$ with a range from 54 to 93\% [33]. In radiological stage I and II sarcoidosis, EBUS-TBNA is also superior to transbronchial lung biopsy, which represented to date the standard diagnostic technique [34].

EBUS-TBNA also plays a role in the diagnosis of TB. In a prospective study, 59 patients with suspected $\mathrm{TB}$ were enrolled. In 41 of the 59 patients, TB diagnosis was confirmed by various diagnostic techniques, and in 35 patients it was established by EBUS-TBNA. Thus, EBUSTBNA had a sensitivity of $85 \%$ [35].

In summary, EBUS has emerged as important diagnostic tools in various pulmonary disorders. EBUS provides a guidance system for TBB of peripheral lesions as well as for TBNA of hilar and mediastinal lymph nodes or masses. Thus, EBUS-guided TBB and EBUS-guided TBNA contribute significantly to the diagnosis of benign as well as malignant diseases. As EBUS-TBNA and EUSFNA present minimally invasive procedures with high sensitivity and high negative predictive value, it is recommended as the first step for mediastinal staging in lung cancer patients. Furthermore, EBUS enables the evaluation of tumor invasion of the tracheobronchial wall and influences therapy planning in early lung cancer as well as in advanced malignancies.

\section{References}

1 Ziegler K, Sanft C, Semsch B, et al: Endosonography is superior to computed tomography in staging tumors of the esophagus and cardia. Gastroenterology 1988;94(suppl):A517.

$>2$ Hürter T, Hanrath P: Endobronchial sonography in the diagnosis of pulmonary and mediastinal tumors. Dtsch Med Wochenschr 1990;115:1899-1905.

-3 Hürter T, Hanrath P: Endobronchial sonography: feasibility and preliminary results. Thorax 1992;47:565-567.

$\checkmark 4$ Wisnivesky JO, Yung RC, Mathur PN, Zulueta JJ: Diagnosis and treatment of bronchial intraepithelial neoplasia and early lung cancer of the central airways: diagnosis and management of lung cancer, 3rd ed: American College of Chest Physicians evidence-based clinical practice guidelines. Chest 2013;143(suppl 5):e263S-e277S.

5 Sun J, Garfield DH, Lam B, Yan J, Gu A, Shen J, Han B: The value of autofluorescence bronchoscopy combined with white light bronchoscopy compared with white light alone in the diagnosis of intraepithelial neoplasia and invasive lung cancer: a meta-analysis. J Thorac Oncol 2011;6:1336-1344.

6 Vincent BD, Fraig M, Silvestri GA: A pilot study of narrow-band imaging compared to white light bronchoscopy for evaluation of normal airways and premalignant and malignant airways disease. Chest 2007;131:17941799.

7 Sutedja G, Golding RP, Postmus PE: High resolution computed tomography in patients referred for intraluminal bronchoscopic therapy with curative intent. Eur Respir J 1996;9: 1020-1023.

8 Becker HD, Herth F: Endobronchial ultrasound of the airways and the mediastinum; in Bolliger CT, Mathur PN (eds): Interventional Bronchoscopy. Prog Respir Res. Basel, Karger, 2000, vol 30, pp 80-93. 
>9 Miyazu Y, Miyazawa T, Kurimoto N, Iwamoto Y, Kanoh K, Kohno N: Endobronchial ultrasonography in the assessment of centrally located early-stage lung cancer before photodynamic therapy. Am J Respir Crit Care Med 2002; 165:832-837.

10 Takahashi H, Sagawa M, Sato M, Sakurada A, Endo C, Ishida I, Oyaizu T, Nakamura Y, Kondo T: A prospective evaluation of transbronchial ultrasonography for assessment of depth of invasion in early bronchogenic squamous cell carcinoma. Lung Cancer 2003;42: 43-49.

$>11$ Herth FJF, Becker HD, LoCicero J, Ernst A: Endobronchial ultrasound improves classification of suspicious lesions detected by autofluorescence bronchoscopy. J Bronchol 2003; 4:249-252.

-12 Herth F, Ernst A, Schulz M, Becker H: Endobronchial ultrasound reliably differentiates between airway infiltration and compression by tumor. Chest 2003;123:458-462.

13 Wakamatsu T, Tsushima K, Yasuo M, Yamazaki Y, Yoshikawa S, Koide N, Fujimori M, Koizumi T: Usefulness of preoperative endobronchial ultrasound for airway invasion around the trachea: esophageal cancer and thyroid cancer. Respiration 2006;73:651-657.

-14 Rivera MP, Mehta AC, Wahidi MM: Establishing the diagnosis of lung cancer: diagnosis and management of lung cancer, 3rd ed: American College of Chest Physicians evidence-based clinical practice guidelines. Chest 2013;143:e142S-e165S.

$>15$ Eberhardt R: EBUS guidance for peripheral biopsy; in Ernst A, Herth FJF (eds): Principles and Practice of Interventional Pulmonology. New York, Springer, 2013, pp 205-215.

$>16$ Herth FJF, Becker HD: Endobronchial ultrasound-guided transbronchial lung biopsy in solitary pulmonary nodules and peripheral lesions. Eur Respir J 2002;20:972-974.

-17 Steinfort DP, Khor YH, Manser RL, Irving LB: Radial probe endobronchial ultrasound for the diagnosis of peripheral lung cancer: systematic review and meta-analysis. Eur Respir J 2011;37:902-910.
18 Tay JH, Irving L, Antippa P, Steinfort DP: Radial probe endobronchial ultrasound: factors influencing visualization yield of peripheral pulmonary lesions. Respirology 2013;18:185190.

19 Asahina H, Yamazaki K, Onodera Y, Kikuchi E, Shinagawa N, Asano F, Nishimura M: Transbronchial biopsy using endobronchial ultrasonography with a guide sheath and vir tual bronchoscopy navigation. Chest 2005; 128:1761-1765

20 Schuhmann M, Bostanci K, Bulgalho A, Warth A, Schnabel PA, Heth FJ, Eberhardt R: EBUS guided cryo biopsies in peripheral pulmonary lesions - a feasibility study. Eur Respir J 2013, E-pub ahead of print.

21 Schiepatti E: La puncion mediastinal a traves deI espolon traqueal. Rev As Med Argent 1949;663:497-499.

22 Oho K, Kato H, Ogawa I, et al: A new needle for transfiberoptic bronchoscope use. Chest 1979;76:492.

23 Wang KP, Terry PB: Transbronchial needle aspiration in the diagnosis and staging of bronchogenic carcinoma. Am Rev Respir Dis 1983; 127:344-347.

24 Yasufuku K: Linear endobronchial ultrasound; in Ernst A, Herth FJF (eds): Principles and Practice of Interventional Pulmonology. New York, Springer, 2013, pp 185-195.

25 Uemura S, Yasuda I, Kato T, Doi S, Kawaguchi J, Yamauchi T, Kaneko Y, Ohnishi R, Suzuki T, Yasuda S, Sano K, Moriwaki H: Preoperative routine evaluation of bilateral adrenal glands by endoscopic ultrasound and fine-needle aspiration in patients with potentially resectable lung cancer. Endoscopy 2013; 45:195-201.

26 Herth FJ, Krasnik M, Kahn N, Eberhardt R, Ernst A: Combined endoscopic-endobronchial ultrasound-guided fine-needle aspiration of mediastinal lymph nodes through a single bronchoscope in 150 patients with suspected lung cancer. Chest 2010;138:790-794.

27 Hangwbo B, Lee GK, Lee HS, Lim KY, Lee SH, Kim HY, Lee HS, Kim MS, Lee JM, Nam BH, Jo JI: Transbronchial and transesophageal fine-needle aspiration using an ultrasound bronchoscope in mediastinal staging of potentially operable lung cancer. Chest 2010; 138:795-802.
28 Silvestri GA, Gonzales AV, Jantz MA, Margolis ML, Gould MK, Tanoue LT, Harris LJ, Detterbeck FC: Methods for staging non-small cell lung cancer: diagnosis and management of lung cancer, 3rd ed: American College of Chest Physicians evidence-based clinical practice guidelines. Chest 2013;143:e211Se250S.

29 Yasufuku K, Nakajima T, Motoori K, Sekine Y, Shibuya K, Hiroshima K, Fujisawa T: Comparison of endobronchial ultrasound, positron emission tomography, and CT for lymph node staging of lung cancer. Chest 2006;130: 710-718.

30 Annema JT, van Meerbeeck JP, Rintoul RC, et al: Mediastinoscopy versus endosonography for mediastinal nodal staging of lung cancer. A randomized trial. JAMA 2010;304:22452252.

-31 Yasufuku K, Pierre A, Darling G, de Perrot M, Waddell T, Johnston M, da Cunha Santos G, Geddie W, Boerner S, Le LW, Keshavjee S: A prospective controlled trial of endobronchial ultrasound-guided transbronchial needle aspiration compared with mediastinoscopy for mediastinal lymph node staging of lung cancer. J Thorac Cardiovasc Surg 2011;142:13931400.

32 Tournoy KG, Keller SM, Annema JT: Mediastinal staging of lung cancer: novel concepts. Lancet Oncol 2012;13:e221-e229.

33 Agarwal R, Srinivasan A, Aggarwal AN, Gupta D: Efficacy and safety of convex probe EBUS-TBNA in sarcoidosis: a systematic review and meta-analysis. Respir Med 2012; 106:883-892.

34 Oki M, Saka H, Kitagawa C, Kogure Y, Mutara N, Ichihara S, Moritani S: Prospective study of endobronchial ultrasound-guided transbronchial needle aspiration of lymph nodes versus transbronchial lung biopsy of lung tissue for diagnosis of sarcoidosis. J Thorac Cardiovasc Surg 2012;143:1324-1329.

35 Sun J, Teng J, Yang H, Li Z, Zhang J, Zhao H, Garfield DH, Han B: Endobronchial ultrasound-guided transbronchial needle aspiration in diagnosing intrathoracic tuberculosis. Ann Thorac Surg 2013, E-pub ahead of print. 